潘玮 ${ }^{1,2}$, 汪静莹 ${ }^{1,2}$, 刘天俐 ${ }^{3}$, 刘晓倩 ${ }^{1}$, 刘明明 ${ }^{1,2}$, 胡斌 ${ }^{4}$, 朱廷劯 ${ }^{1 *}$

1. 中国科学院心理研究所, 北京 100101 ;

2. 中国科学院大学心理学系, 北京 100049;

3. 北京大学人口研究所, 北京 100871 ;

4. 兰州大学信息科学与工程学院, 兰州 730000

* 联系人, E-mail: tszhu@psych.ac.cn

2018-04-02 收稿, 2018-05-16 修回, 2018-05-18 接受, 2018-07-11 网络版发表

摘要抑郁症是世界范围内常见的精神疾病之一, 抑郁症患者往往长期伴随情绪低落, 如悲伤内疚、低自尊、 兴趣丧失、功能减退等，对个人、家庭及社会造成了巨大损失。抑郁症的发病原因复杂，临床诊断存在一定的困难， 有必要寻找一种更加便捷、客观、高效的方式来辅助抑郁症的快速识别. 语音作为一个相对客观且容易获得的变 量, 具有其潜在的价值. 本研究旨在构建基于语音的抑郁症识别模型, 探究语音与抑郁症之间的关系. 收集了103 名被试(45名抑有症患者, 58 名健康人)的语音数据, 实验组为临床确诊的抑郁症患者, 年龄在 23.8 44.6岁之间, 控 制组为健康人，年龄为20.1 41.7岁。我们采用了 3(情绪状态：正性、中性、负性) $\times 3$ (任务类型：语言问答、文本朗 读、图片描述)的实验设计, 运用机器学习的分类算法——逻辑回归(LR)来构建抑郁识别模型. 实验结果表明, 语 音的抑郁识别精度可以达到 $82.9 \%$. 本文采用机器学习方法, 基于语音变量建立有效的抑郁症自动识别模型, 为 抑郁症的辅助识别提供客观的指标和依据.

关键词抑郁症, 语音特征, 分类算法, 逻辑回归

世界卫生组织(World Health Organization)2017 年的数据显示, 全球有超过 3 亿人患有抑有症, 且这 一数字在逐年增加. 调查显示, 中国有 5400 多万抑郁 症患者. 抑郁症不仅影响患者的身心健康, 更会导致 高致残致死率. 有报告指出, 抑郁症患者的自杀率是 普通人群自杀率的 20 倍以上, 且在已自杀者中, 抑郁 症患者占 $1 / 2$ 以上 ${ }^{[1]}$. 抑郁症不仅给个人和家庭带来 巨大的创伤，其后果对社会也是一种巨大的损失.

抑郁症虽然危害巨大, 但它是一种可以被有效 治疗和改善的疾病. 临床实践中, 药物治疗能够促使 抑郁症患者康复, 此外还有心理治疗 (如认知行为疗 法、人际心理疗法、行为治疗、精神动力学治疗) 以 及物理治疗 (如重复经颖磁刺激)等. 然而有相当一部
分患者群体没有得到及时的诊断. 国内外受抑郁症 影响的人群中有不到 $1 / 2$ (在许多国家，只有不到 $10 \%$ ) 接受治疗 ${ }^{[1]}$. 这一现象的原因大致有以下 3 点.

( i ) 长期以来, 抑郁症被公众所误解, 常常被 贴上“小心眼”“想不开”“矫情”的标签而缺少足够的 重视. 事实上, 抑郁症是一种严重的心境障碍. 精 神疾病, 往往同其他心理疾病一样, 公众缺乏足够的 认识. 躯体疾病者求医会被认为是正常的, 而心理疾 病，尤其是精神疾病者，会被大众贴上“精神不正常” 的标签. 社会的高速发展、高强度的工作压力、社会 身份的变更、人际关系甚至躯体病患的影响，都会增 加抑郁症患病的可能. 20 60岁是抑郁症的高发期, 青春期、职业人群、更年期以及老年阶段都是抑郁症 
的高发阶段. 抑郁症涉及人群广泛, 可能引发严重后 果, 应该引起足够的重视.

(ii) 抑郁症患者求助意愿不够强烈. 一方面, 抑有症患者各项功能受损, 情绪体验较为消极, 兴趣 减退, 缺乏积极改善现状的愿望. 另一方面, 抑郁症 患者对抑郁症的认识不够, 更倾向于隐藏自己患病 的事实, 讳疾忌医. 《2017年中国网民抑郁症调研报 告》显示, 当个体出现抑郁情绪时, 仅 $5 \%$ 的人表示会 寻求专业机构或个人的帮助, 而其他 $95 \%$ 则选择忍 耐或者跟亲友倾诉.

（iii）相关资源的缺乏及医疗条件的限制. 一方 面, 由于对精神疾病的重视不够, 相应的资金投人和 专业医疗人士、心理服务工作者相对缺乏; 另一方面, 对抑郁症的有效篮查存在一定难度, 目前对抑郁症 的诊断往往由专门的精神科医生借助精神疾病诊断 与统计手册(the diagnostic and statistical manual of mental disorders, DSM)进行诊断, 该手册的使用需要 经过严格训练, 测试时间往往较长, 并且应用场景也 受限, 与此同时, 还存在量化不够精确等问题 ${ }^{[2,3]}$. 此外, 对抑郁症的诊断存在误诊情况. 例如, 抑有症 患者往往伴随躯体不适, 因此他们会更多以躯体不 适为主诉到综合医院就诊, 抑郁症患者到内科的就 诊率远远高于到精神科的就诊率. 而综合医院的医 生往往相对缺乏精神疾病诊断的临床经验. 即便是 精神科医生, 在诊断过程中也难免会带有一定的主 观倾向, 诊断的效力相对较低. 再者, 抑郁症与双相 障碍是两种比较类似的心境障碍类疾病, 抑郁症和 双相障碍的抑有期往往较难区分 ${ }^{[4]}$. 《全球疾病负担》 (Global Burden of Disease, GBD)在2016年的多项针 对中学生的流行病学的调查发现 ${ }^{[5]}$, 中学生抑郁症状 的检出率为 $23.7 \%$ 54.4\%, 其中重度抑有症状检出率 为 $3.3 \% \sim 9.68 \%$.

抑郁症治疗的关键在于前期的诊断篮查, 如果 能够在相对安全、不过多涉及隐私的情况下快速有效 地诊断出个体是否患有抑郁症, 将极大地降低抑郁 症临床篮查的困难, 促使患者尽早接受治疗. 因此, 有必要引人更加客观便捷的测量方式, 打破传统医 学诊断的限制, 以更加灵活高效的方式辅助临床抑 郁症的早期篮查, 帮助患者得到及时有效的治疗.

语音是一种非侵人式、临床极易获取的信息. 目 前已有关于语音与抑郁症的大量研究, 为探索语音 作为临床抑郁症自动化诊断的工具提供了可能. 根
据已有研究, 抑有症病人的语音具有以下特点: 语速 较慢、停顿次数多、停顿时间长 ${ }^{[6,7]}$ 、语音特征的变 化减少 ${ }^{[8]}$ 、声音缺少抑扬顿挫、呆板 ${ }^{[9]}$. 抑有组个体与 正常个体相比, 气息声更显著 ${ }^{[10]}$. 从韵律特征的角度 来看, 抑有症患者的基频(fundamental frequency, F0) 变化减少, 如带宽、振幅、能量等 ${ }^{[6,7,11]}$, 反映了抑郁 症患者声音频率的变化变少. 频谱特征也与患者的 抑郁程度有关, 有研究发现, $500 \mathrm{~Hz}$ 以下和 500 $1000 \mathrm{~Hz}$ 的声谱能量的变化程度和抑郁的严重程度增 加相关 ${ }^{[12]}$. 可见, 对语音进行特征抽取, 对相对客观 且不容易被个体刻意掩盖的声学特征的捕捉, 将有 助于更好地理解抑郁症. 针对抑郁症患者语音特点 的研究大致有以下两大类.

( i ) 追踪研究. 早期阶段, 研究者已经发现生 理或者心理上的轻微变化会导致声音特征的显著改 变 ${ }^{[13]}$. 心理疾病, 或者说精神疾病会伴随病人声音 特性(voice)和语音特征(speech, 包括词汇语法)等的 改变 ${ }^{[14,15]}$. 针对精神疾病患病群体的声学分析发现, 言语相关变量与症状测评结果存在着中等以上的相 关 ${ }^{[16,17]}$. 在这一阶段, 研究者主要进行追踪研究, 借 助于治疗前后患者语音特点的变化情况进行探索. 例如, Darby和Hollien ${ }^{[18]}$ 最早提出借助实验方法考察 精神疾病对病人言语的影响, 采用7种语音维度组成 的语音声音量表, 结合前后测的方式, 发现抑郁症病 人在治疗前后的声音有显著不同. 但是, 在这一类研 究中, 存在追踪时间较短, 被试数量比较少或缺乏对 照等问题. 例如, Szabadi等人 ${ }^{[19]}$ 对 8 名被试(4名健康 人和 4 名中度抑郁症患者)进行了两个月的追踪研究, 结果发现, 停顿时间的增长可以作为精神运动阻滞 的客观指标, 而精神运动阻滞是抑郁症的重要特征 之一. Mundt等人 ${ }^{[6]}$ 对 35 名抑郁症患者进行了持续6周 的电话访谈收集被试的语音. 抑郁症患者的治疗过 程往往较长, 并且患者在这一过程中可能存在病情 不稳的情况, 此外, 较少的被试量以及特殊的数据收 集方式, 如电话访谈, 都可能会影响数据结果的说服 力及推广性.

(ii) 横向研究. 比较抑郁症患者群体与其他不 同群体之间的语音特点. 例如, Flint等人 ${ }^{[20]}$ 对比了重 度抑郁症、帕金森患者与正常人语音特点的异同, 结 果发现, 两类病人与正常人相比, 发音起始时间 (voice onset time)更短, 第二共振峰迁移(second formant transition)减少. France等人 ${ }^{[9]}$ 对比了正常人、抑 
郁患者和有自杀倾向的人的语音特点, 结果发现共 振峰(formant)和功率谱密度 (power spectral density) 特征在分类问题中是有效特征. 另有研究发现, 抑郁 患者比正常人在语音上缺少韵律 (prosody) 变化 ${ }^{[21]}$. 此外还有一些跨文化的有关研究. 例如, Alghowinem 等人 ${ }^{[22]}$ 对美国、德国、澳大利亚三个国家的两个语 种(英语和德语)进行了实验, 结果发现语言种类和文 化并不能显著影响结果的好坏. 鉴于这些研究的实 验设计都相对简单. 一些研究开始设计更加复杂的 任务来验证语音与抑郁症之间的关联是否具有跨任 务的一致性. 例如, Alghowinem等人 ${ }^{[23]}$ 指出自发语 音的分类准确率比文本朗读的高. Mitra等人 ${ }^{[24]}$ 的研 究显示, 自然语音要比朗读获取的语音对是否抑郁 的预测效果更好. 可见, 言语方式也会影响到实验的 结果. 不同的言语方式表明说话人在信息收集、认知 加工、言语组织等过程中存在差异, 这一点可能会反 映在语音特征的变化上, 进而影响了实验结果.

此外, 还有从特征选取和算法改进方面来研究 如何提高语音对抑有症的预测效果 ${ }^{[25 ~ 27]}$.

更加复杂的实验设计使得实验结果更加具有说 服力. 但是, 这些研究往往偏向于探索通过算法改进 来提高语音对抑有症的预测效果, 这些针对特定数 据集的算法改进较少得到有效的重复性验证. 且这 些研究较少对不同任务取得的不同结果进行更进一 步的探讨. 有研究发现, 与健康人相比, 重度抑郁症 患者对正性情绪(如喜悦)的反应减弱, 对负性情绪 (如悲伤、恐惧、愤怒)的反应增强, 且这一偏差与抑 郁症患者的病情严重程度与功能失调症状程度相 关 ${ }^{[28]}$. 另有研究发现, 抑郁症患者对积极的人脸图 片反应减弱 ${ }^{[29]}$, 而对消极的面部表情反应增强, 并 且对消极表情的记忆更好 ${ }^{[30]}$. 这些研究表明, 抑郁 症患者对不同效价的情绪刺激的反应出现钝化现象. 情绪低落是抑郁症患者的主要特点之一. Stasak等 人 $^{[31]}$ 的研究发现, 增加情绪效价这一信息能够将语 音特征对是否抑郁的预测准确率提高 $5 \%$. 可见, 发 音与情绪之间也存在着一定的关联. 如果能够同时 考虑情绪效价和任务类型两种因素, 将能够更加深 人地了解个体的发音特点及其与情绪的关联, 并对 结果进行更好的解释. 与此同时, 也能够进一步验证 不同任务类型与不同情绪状态下语音对抑郁症的预 测效果是否一致.

为了考察研究结果是否具备跨任务的稳定性,
我们创设了 3 种实验情景：语言问答、文本朗读和图 片描述. 为了考察不同情绪状态下, 语音特征对抑郁 症的预测效果是否一致, 本研究准备了 3 种效价的情 绪启动材料, 旨在启动个体的不同情绪状态, 获得不 同情绪状态下的语音. 同时, 本文旨在通过提取语音 特征, 建立针对抑郁症辅助识别的预测模型, 提高对 抑郁症的有效诊断. 目前国内有关研究正处于起步 阶段, 本文将探究在不同任务和不同情绪状态下, 语 音变量对抑郁症的预测效果, 探索语音作为临床抑 郁症诊断辅助工具的潜在价值.

\section{1 方法}

\section{1 数据采集}

本研究共包含 103 名被试. 其中, 抑郁症患者来 自北京安定医院与回龙观医院, 健康人由广告招募 而来. 所有被试均经过经验丰富的心理专家和精神 科医生依照《简明国际神经精神访谈》(the MINIInternational Neuropsychiatric Interview, MINI) ${ }^{[32]}$ 和 《心理障碍诊断与统计手册》(Diagnostic and Statistical Manual of Mental Disorders, DSM-IV) $)^{[33]}$ 进行诊 断䇻查. 实验招募了 45 名抑郁症患者与 58 名健康人, 前者年龄在23.8 44.6岁 $(M=34.2, S D=10.4)$; 后者年 龄为 $20.1 \sim 41.7$ 岁 $(M=30.9, S D=10.8)$. 从性别角度来 看, 抑郁症患者中, 男性为 22 人, 女性为 23 人; 健康 人中, 男性共 27 人, 女性共 31 人. 所有被试不存在物 质滥用、物质依赖、人格障碍等其他精神疾病, 无 严重的躯体疾病或自杀行为. 被试为小学以上文化 水平.

本研究为 3 (情绪状态: 正、中、负) $\times 3$ (任务类型： 语言问答、文本朗读、图片描述)的实验设计. 其中, 语言问答任务的 3 种情绪条件下各有3道相同情绪效 价的问题, 文本朗读任务在各种情绪下各有一段文 本材料, 图片描述任务中, 每种情绪下分别有两张图 片, 一张为人脸图片, 一张为情景图片.

实验在光线充足、安静的房间内进行. 所有任务 与指导语都在同一电脑程序中, 被试坐在距离一台 21 寸电脑屏幕正前方约 $1 \mathrm{~m}$ 远的地方, 屏幕中会显示 任务要求. 每一任务材料呈现后, 被试需要根据提示 进行回答, 主试在一旁对程序进行操作, 并使用录音 设备记录被试的语音. 在语言问答任务中, 被试需要 根据播放的问题进行回答. 例如，在正性情绪启动条 
件下, 问题为: “请跟我们分享一段您认为美好的回 忆, 大致描述下当时的情景.” 文本朗读任务中, 屏 幕中将显示一段文本, 被试需要仔细汶览一遍文本, 之后进行朗读. 图片描述任务中, 屏幕中将显示一张 图片和一句提示语: “该图片让你联想到什么?”被试 需根据图片和提示进行联想并进行语言描述. 具体 说明见补充材料.

所有任务随机呈现且仅呈现一次. 所有材料均 来自现有文献并且具有显著启动效果, 语音问答任 务为被试根据不同的任务要求进行回答 ${ }^{[34,35]}$. 在此 过程中对被试的声音进行录音, 从而获得语音数据. 每个被试在每种条件下分别生成一份语音数据. 本 研究经北京安定医院和回龙观医院伦理委员会审批 并获得许可. 实验前被试均签署知情同意书.

\section{2 数据处理}

数据以.wav形式进行保存, 在此基础上进行特 征提取. 结合前人研究, 我们选取了 26 个在抑郁症研 究中应用较为广泛的语音特征作为研究对象, 包括强 度 (intensity)、响度 (loudness)、过零率(zero-crossing rate)、清浊比率(voicing probability)、基频、基频包络 (F0 envelope)、8个线性谱对(line spectral pairs, LSP) 以及 12 个梅尔倒谱系数 (mel-frequency cespstral coefficients, MFCC). 其中, 强度为声波在单位时间内作 用在与其传递方向垂直的单位面积上的能量. 响度 即音量, 由振幅决定. 过零率指信号的符号变化(如 从正变到负)的比率. 清浊比率用于评估每个调波中 清音和浊音能量的百分比. 基频指一段周期性声波 的最低频率. 基频包络是与音色有关的基频特征. 线 性频谱对是指声音通过频道传输时的线性预测系数. 而梅尔倒谱系数, 是基于声音频率其非线性梅尔刻
度 (melscale) 的对数能量频谱的线性变换的系数 ${ }^{[36]}$. 在这些静态特征的基础上, 计算了能够反映语音动 态变化的这 26 个特征的一阶导数(delta value), 并进 一步获得了能够反映语音整体变化的 19 个长时特征, 包括最大值、最小值、全距、均值、标准差、峰度和 偏度等统计指标. 采用专门的特征提取软件 openSMILE ${ }^{[36]}$, 一共获得了 988 个语音特征.

其中, 短时特征的提取过程为按照帧移 (frame step) $10 \mathrm{~ms}$, 帧长(frame size) $25 \mathrm{~ms}$ 将音频文件分帧, 可分为 498 帧. 对于不同的韵律特征，有两种不同的 方式来进一步提取. 例如, 对于基频、基频包络、清 浊比率三个特征, 需要进一步使用汉明窗 (Hamming) 函数处理信号, 减少帧间信号的不连续性, 随后进行 快速傅里叶变换(fast Fourier transform, FFT) 以便从 频域处理信号, 应用自相关函数 (autocorrelation function, ACF) 求得浊音的基音周期, 从每一帧信号 中得到对应这三个短时特征(基频、基频包络、浊音 概率), 如图 1 所示. 对于其他 23 个特征, 在音频文件 被分为498帧之后, 对语音的高频部分进行预加重 (pre-emphasis), 去除口唇辐射的影响, 增加语音的 高频分辨率, 接着通过汉明窗运算、快速傅里叶变换 和梅尔倒谱分析, 可以得到 12 个梅尔倒谱系数. 基于 自回归模型, 即 AR(auto-regressive)模型, 进行线性 预测编码(linear predictive coding, LPC). 之后从每一 帧信号中得到其余 11 个短时特征(如强度、响度、过 零率、线性频谱对等). 流程如图2所示.

对于长时特征(统计特征), 对每一帧获得的 26 个 短时特征进行平滑处理和一阶导数运算, 得到 26 个短 时Delta特征, 至此, 每一帧共有 52 个短时特征. 对每 一个短时特征, 在 498 帧上纵向进行统计运算, 如对 基频(F0)这个短时特征，在所有帧上取最大值(max)和

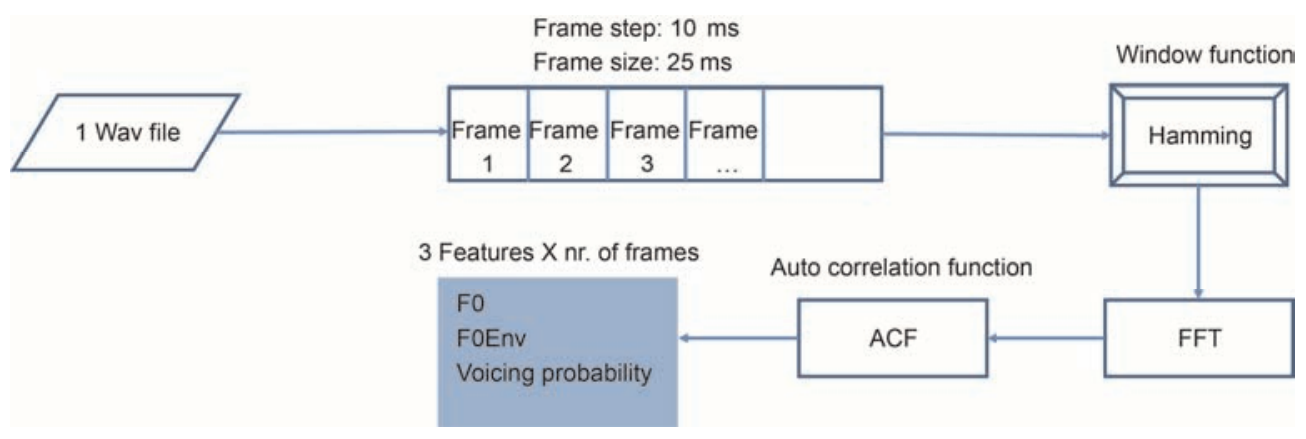

图 1 (网络版彩色) 3 个短时语音特征提取流程

Figure 1 (Color online) Extracting procedure for 3 short-term voice features 


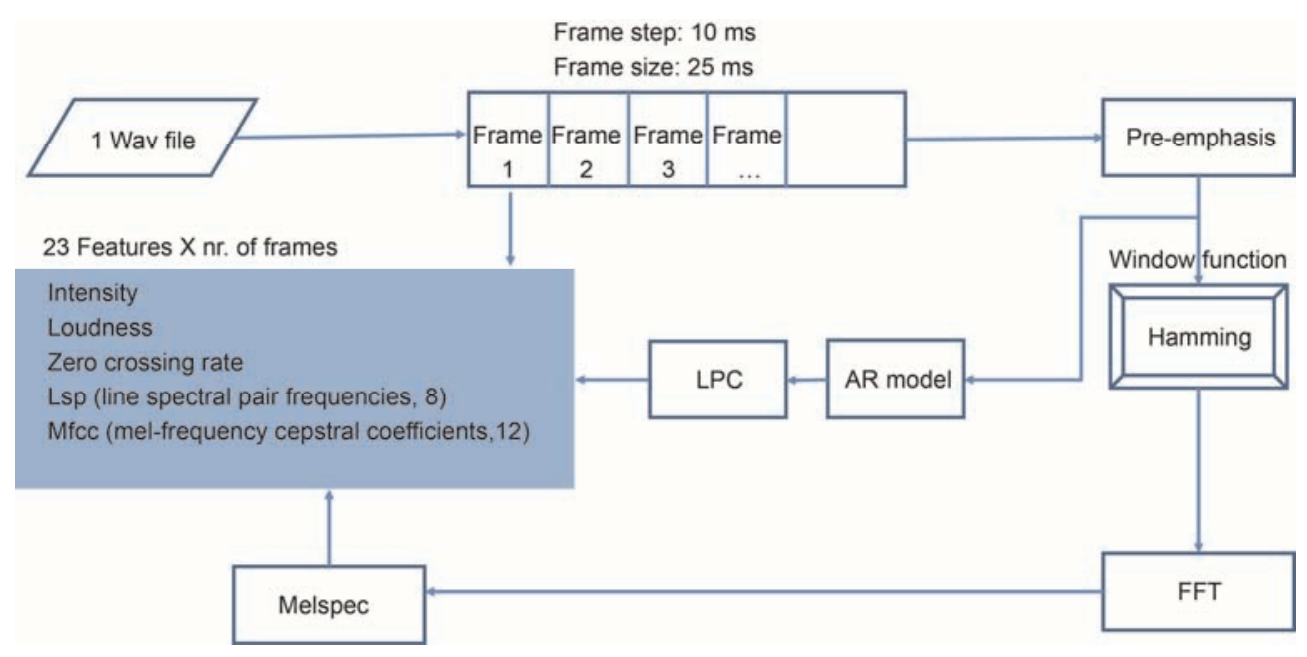

图 2 (网络版彩色) 23 个短时语音特征提取流程

Figure 2 (Color online) Extracting procedure for 23 short-term voice features

最小值(min), 得到两个统计特征(长时特征). 按照上 一步的思路, 对 52 个特征, 分别在所有帧上进行 19 种 统计运算, 最后一个音频文件共得到 988 个长时特征. 具体处理流程如图3所示.

\section{3 模型构建}

本研究利用WEKA ${ }^{[37]}$ 软件构建抑郁症分类模型, 借助于逻辑回归(logistic regression)分析方法建立语 音的预测模型.

逻辑回归 ${ }^{[38]}$ 是线性回归的另一种形式, 线性回 归用于对连续变量进行预测. 当需要对二分变量以 及较复杂现象进行预测时, 就可以运用逻辑回归方 法. 其核心是通过将数据拟合到logit函数中, 从而对 事件发生的概率进行预测. 它是一种类似于朴素贝
叶斯的算法, 逻辑回归的限制较前者宽松. 且该方法 更加适用于复杂数据的分类问题.

\section{4 评估指标}

分类结果的好坏需要有评价指标与标准. 机器 学习的分类算法中有以下几个分类结果好坏的评估 指标：准确率(precision)也称真阳性率, 也就是信号 检测论中的击中/(击中+虚报) ${ }^{[39]}$; 召回率(recall)相当 于击中率 ${ }^{[39]}$; F值(F score) 是准确率与召回率的加权 调和平均数 ${ }^{[39]}$.

曲线下面积(area under curve, AUC): 受试者工 作特征曲线(receiver operating characteristic curve, ROC曲线)下面积, AUC是二分类模型常用的评价指 标 $^{[40]}$. 指给定一个正样本和一个负样本, 分类器输

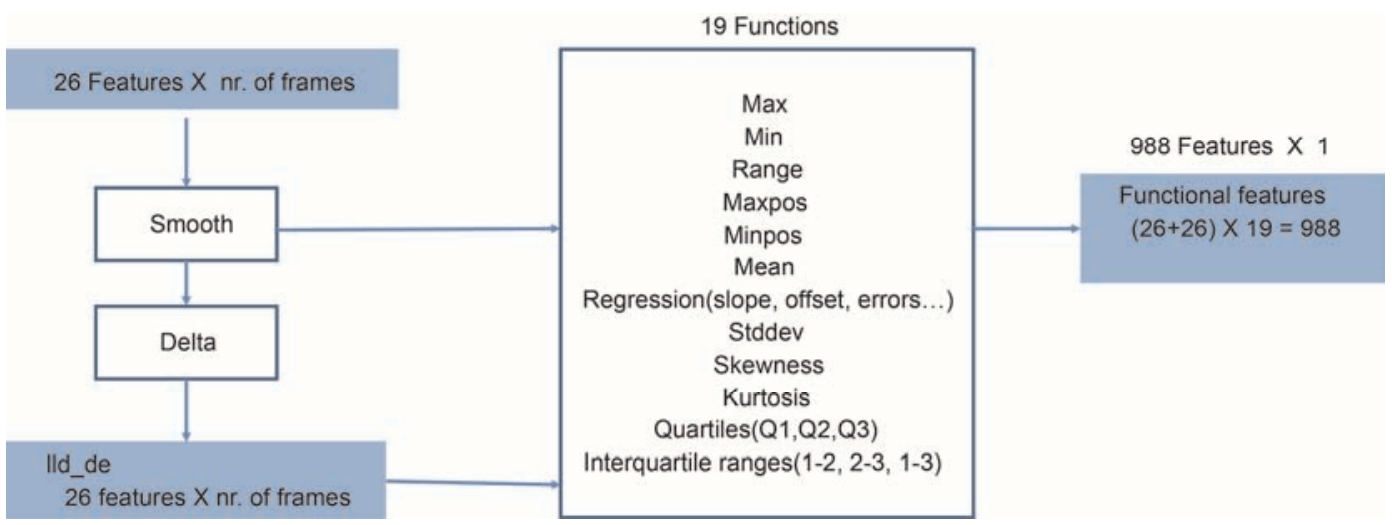

图 3 (网络版彩色) 26 个语音对应的 988 个长时特征提取流程

Figure 3 (Color online) Extracting procedure for 988 long-term voice features 
出正样本为正的概率比分类器输出负样本为正的概 率要大的可能性. AUC越大, 表明分类模型的预测准 确率越高, 分类器效果更好, 诊断工具的价值越大.

\section{2 结果}

借助逻辑回归算法, 分类预测结果显示, 在正性 情绪启动下, 语音问答任务的预测效果最好, 其预测 准确率为 $77.3 \%$, 召回率为 $73.9 \%$; 在中性情绪启动 下, 图片描述任务的效果最好, 其预测准确率为 $76.1 \%$, 召回率为 $74.5 \%$; 负性材料中, 语音问答的效 果最好, 其预测准确率为 $82.9 \%$, 召回率为 $73.9 \%$. 以 中性情绪启动下图片描述任务的结果为例进行说明, 在该条件下的预测模型中, 所有被预测为抑有的人 中, 临床确诊为抑郁症的比例为 $76.1 \%$; 在所有临床 确诊为抑郁症的人中, 被预测为抑郁的比例为 $74.5 \% . F$ 值是准确率与召回率的权衡. 在正性情绪启 动下, 语音问答任务下的分类预测模型要优于文本 朗读任务下的预测模型, 而后者又优于图片描述任 务中的预测模型 $(F$ 值: 75.6>66.7>62.9). 在中性情绪 启动下, 图片描述任务中的预测模型要优于文本朗 读任务结果, 而文本朗读任务下模型结果与语音问 答任务下结果相近 $(F$ 值: $75.3>62.4>60.2)$; 在负性情 绪启动下, 语音问答任务的预测效果最理想, 文本朗 读任务下的预测模型效果次之, 图片描述任务下结 果最差 ( $F$ 值: $78.2>68.9>60.7$ ). 从图4可以看出, 在语 音问答任务中的负性情绪材料启动下, $F$ 值最大, 为 $78.2 \%$. 在所有条件下, $F$ 值在 $60.2 \%$ 78.2\%.

在语音问答任务中, 负性材料的预测效果最好, 准确率为 $82.9 \%$, 召回率为 $73.9 \%$, 其次是正性材料, 其预测准确率为 $77.3 \%$, 召回率为 $73.9 \%$, 然后是中 性材料, 准确率为 $67.6 \%$, 召回率为 $54.3 \%$. 在文本朗 读任务中, 类似的, 负性和正性材料的预测效果较
好, 预测准确率分别为 $67.4 \%$ 和 $66 \%$, 召回率分别为 $70.5 \%$ 和 $67.4 \%$. 中性材料的预测效果最差, 预测准 确率为 $61.7 \%$, 召回率为 $63 \%$. 但是在图片描述任务 中, 中性材料的预测效果最好, 准确率为 $76.1 \%$, 召 回率为 $74.5 \%$. 而正性和负性材料的预测效果相对较 差, 二者预测准确率分别为 $60.9 \%$ 和 $58.7 \%$, 召回率 分别为 $65.1 \%$ 和 $62.8 \%$. 同样地, $F$ 值与 AUC得分表现 出了同样的分布特点, 例如, 在语音问答任务中, 负 性情绪启动下, 语音特征的抑郁症预测模型效果较 好, $\mathrm{AUC}=0.81$, 曲线下面积这一指标是模型好坏的 重要评价标准, 一般AUC在 $0.5 \sim 1$ 之间, 表明模型分 类效果优于随机猜测, 具有一定的预测价值. 整体来 看, 在不同实验条件下, AUC值在0.66 0.81之间, 均 在 0.6 以上. 具体结果见表 1 及图 4.

整体来看, 在不同的任务及启动条件下, 语音特 征对个体是否抑郁的预测效果基本在 $60 \%$ 以上.

\section{3 讨论}

本研究探讨了语音对抑郁症的预测作用. 结果 显示, 语音数据的分类准确率基本在 $60 \%$ 以上, 即针 对语音数据, 以机器学习的方法建立分类模型, 模型 对个体是否抑郁的预测结果具备跨情景的一致性, 且模型效果较为理想. 这与国外有关语音与抑郁症 的研究结果相一致. Cummins等人 ${ }^{[41]}$ 采用调制的频谱 特征来预测抑郁症, 分类准确率可以达到 $67 \%$. Cohn 等人 ${ }^{[42]}$ 通过提取抑郁患者的语音特征, 如基频和转 换速度(switch duration), 采用留一交叉验证(leaveone-out validation)方式进行逻辑回归分析, 结果表明, 语音特征预测是否抑郁的正确率为 $79 \%$, 且抑郁患 者的F0在治疗后显著下降. 抑有症患者语速的提高、 响度增加、停顿间隔减少已经被认为是比较典型的抑 郁症治疗效果的参数 ${ }^{[43,44]}$. 语音能够预测抑有症这

表 1 不同条件下分类模型预测结果 $(\%)^{\mathrm{a})}$

Table 1 The results of classification models under different conditions (\%)

\begin{tabular}{|c|c|c|c|c|c|c|c|c|c|}
\hline & \multicolumn{3}{|c|}{ 语音问答 } & \multicolumn{3}{|c|}{ 文本朗读 } & \multicolumn{3}{|c|}{ 图片描述 } \\
\hline & 正性 & 中性 & 负性 & 正性 & 中性 & 负性 & 正性 & 中性 & 负性 \\
\hline Precision & 77.3 & 67.6 & 82.9 & 66 & 61.7 & 67.4 & 60.9 & 76.1 & 58.7 \\
\hline Recall & 73.9 & 54.3 & 73.9 & 67.4 & 63 & 70.5 & 65.1 & 74.5 & 62.8 \\
\hline$F$ score & 75.6 & 60.2 & 78.2 & 66.7 & 62.4 & 68.9 & 62.9 & 75.3 & 60.7 \\
\hline AUC & 78.3 & 66.8 & 80.9 & 69.9 & 65.7 & 72.5 & 67.5 & 77.7 & 65.8 \\
\hline
\end{tabular}

a) 从左至右各列数据分别为实验材料编号第 $1,4,7,10,11,12,13,14,18$ 号材料获取的语音数据建立的逻辑回归预测模型. 详见 网络版补充材料 


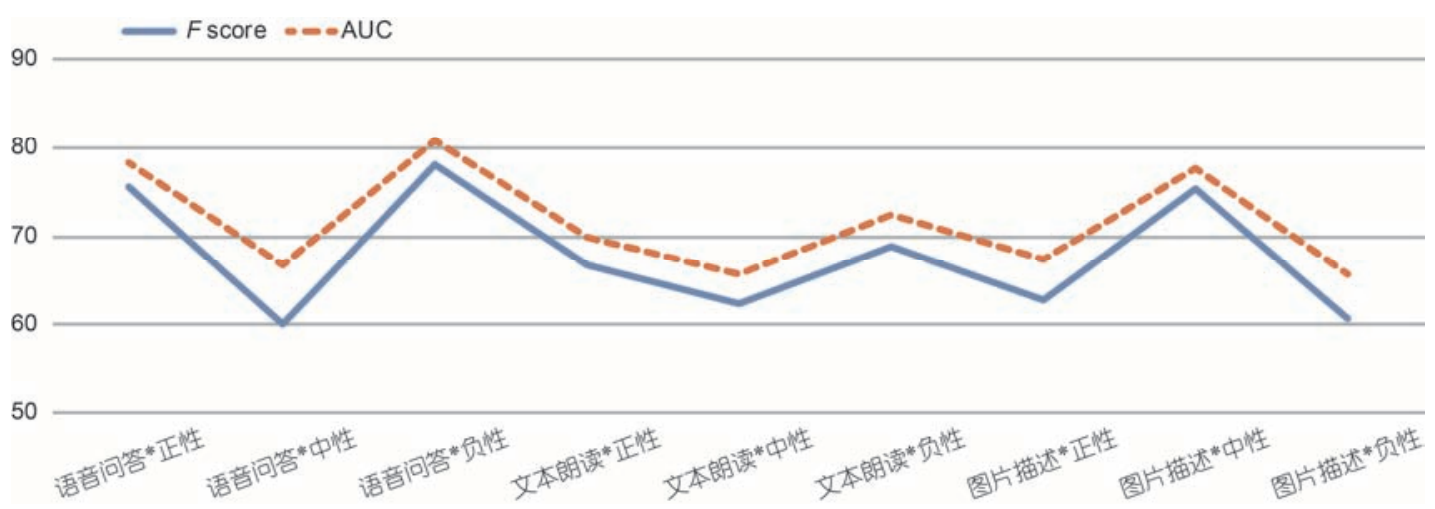

图 4 (网络版彩色) 不同实验条件下分类模型评价指标 $(\%)$

Figure 4 (Color online) Evaluation indexs of classification results under different experimental conditions (\%)

一结论表明，抑有症患者确实存在语音方面的异常.

从情绪的角度来看, 语音特征能够较好地区分 在正中负三种情绪下个体是否患有抑郁症(基本均在 $60 \%$ 以上). 这表明抑郁症患者与正常人相比, 确实 存在情绪方面的异常, 而这一异常体现在了个体的 声音特性上. 脑成像研究发现, 抑有症患者中, 情感 相关的神经系统在结构和功能上均存在异常 ${ }^{[45]}$. 例 如，在杏仁核 ${ }^{[46 ~ 48]}$ 、纹状体 ${ }^{[49 ~ 51]}$ 和背外侧前额叶 ${ }^{[52]}$ 、 前扣带皮层 ${ }^{[53]}$ 、顶叶上回、额眶皮层 ${ }^{[54]}$ 、脑岛 ${ }^{[55]}$ 出 现对负性情绪刺激激活减弱. 这些结论共同说明, 抑 郁症患者在情绪加工方面出现问题. 抑郁症患者在 语音方面与健康人所存在的差异可能是由于抑郁症 患者情绪加工方面出现异常, 而情绪对人体学习、生 活、各项机能等都会有很大的影响, 这一影响就体现 在了个体的声音特性上.

从特征的角度来看, 本文中所提取的语音特征 均为情绪识别中常用的指标. 这进一步说明, 抑有症 患者语音与正常人语音的差异可能更多地反映了抑 郁症患者的情绪状态以及认知方式的不同. 一项针 对自杀行为的研究发现, 抑郁且已经自杀的个体, 与 抑郁但未自杀的个体相比, 其生前情绪状态具有更 多的负性情绪, 如绝望、无助、愤怒、焦虑、被抛弃、 孤独感、羞愧感以及自我厌恶等情感 ${ }^{[56]}$. 根据Beck的 负性自动思维理论(negative independent thought theory $)^{[57]}$, 抑郁症患者更倾向于将正性、中性事件判断为 负性事件, 存在着各种认知偏差, 如选择性注意、负 性过度引申、灾难化、绝对化等认知特点. 这种偏向 性情绪处理障碍可能是抑郁患者处于持续情绪低落 并显现出语音异常的认知方面原因.

从任务的角度看, 正性与负性情绪效价下, 语音
问答任务下模型较理想, 这可能是因为本研究的语 音问答任务是需要被试根据自己的实际情况进行回 答, 个体的以往经历更容易唤起类似的情绪体验, 由 此致使健康人的正性或者负性情绪体验更强烈，与 抑郁患者的反差更大, 故该任务下的模型预测效果 较好. 文本朗读也创设了一个情绪环境, 但是可能没 有个体曾经的经历更能激发自己的情绪体验, 两组 被试的情绪体验差别不大，因此结果没有前者好. 图 片描述由于提供的情绪环境更加受限制, 由此诱发 的抑郁患者和健康人在正性和负性情绪体验上的差 别就更小了. 而在中性情绪启动方面, 可能由于语言 问答和文本朗读给个体创设了过于相似的情景，而 没有任何情绪体验, 故个体的语音特征差异不大, 不 能很好地区分是否抑郁. 但是由于图片描述对个体 的限定比较小, 因此能真实地反映个体在中性情绪 下的语音特征, 这可能暗示了抑郁症患者和健康人 存在认知加工方式的不同，从而使得在面对相同的 图片时产生了不同的情绪体验, 进而反映在了语音 上. 虽然较为理想的结果主要是从实验材料编号第 1 , $4,7,10,11,12,13,14,18$ 这些材料获取的语音数据而 建立的预测模型，这表明并不是每种任务下每个任 务类型都能获得比较好的启动效果, 已有研究发现, 不同的任务可能会导致不同的结果. 例如, Alghowinem等人 ${ }^{[58]}$ 发现, 自发的语音(如访谈、图片描述)比 自动语音(如朗读、数数)能够获得更好的结果, 且悲 伤类的问题对应的语音特征分类效果比其他问题更 好. 这与本研究结果基本一致. 从以往研究来看, 有 研究布置专门的实验场景来收集语音数据 ${ }^{[42]}$, 另有 研究则利用访谈或日常交流过程的录音进行研究 ${ }^{[59]}$. 本研究中, 语音问答任务类似于自然语言访谈任务, 
结合了自然情景下的访谈与实验室语音收集方式, 多角度地考察了不同任务下语音特征对是否抑郁的 预测效果, 能够取得较好的效果, 该研究所建立的的 语音预测模型具有一定的鲁棒性.

从算法模型的角度来看, 本研究所使用的逻辑回 归方法是一种应用非常广泛的机器学习分类算法之一. 它能够利用较少的资源处理大规模数据, 属于比较高 效的一种算法, 具有较好的鲁棒性. 同时, 它又是比 较严谨的分类算法, 通过概率来预测分类结果 ${ }^{[60]}$.

鉴于临床对抑郁症的诊断存在的困难和主观偏 差等问题. 语音作为一个相对客观且容易获得的指 标, 具有一定的优越性. 例如, Alghowinem等人 ${ }^{[61]}$ 采 集了抑郁症患者和健康人的多项指标, 如语音(狭义 的副语言特征(paralinguistic features))、眼睛注视、头 部姿态等, 结果发现语音的预测效果要好于其他特 征. 本研究证实了语音特征可以区分抑有症患者和 健康人, 说明语音这一客观且容易获得的变量在临 床抑郁症诊断的快速识别方面具有的潜在价值, 为 之后的进一步研究奠定了基础.

本研究存在以下不足. 首先, 未来研究需充分考 虑和控制混淆变量, 找出对抑郁症具有高度关联的 协变量, 并做到在实验组和控制组的严格匹配. 这是 因为, 抑郁症是一种病因比较复杂、同时受生物学因 素和社会因素影响的一种复杂的心境障碍疾病. 从 社会的角度来说, 个体的年龄、受教育程度、社会等 级、婚姻状况等因素均可能对个体是否患抑郁具有一 定的影响. 他们之间可能存在着某种关联. 有研究发 现, 首发年龄越早, 自杀倾向、自杀尝试越多, 神经 质越高、睡眠越少、食欲越差、体重变化越大、抑郁 症爆发次数越多、结婚率越低 ${ }^{[62,63]}$. “中国精神障碍疾 病负担及卫生服务利用的研究项目调查” 显示, 抑郁 症患者分居以及离婚率 $(9.47 \%)$ 和丧偶率 $(5.93 \%)$ 均高 于已婚率 $(3.15 \%)$. 小学以下受教育程度者比率为 $3.13 \%$, 大专及以上为 $1.39 \%$. 另有研究发现, 受教育 程度越高, 个体患抑郁症的概率更大, 而受教育程度 低的患者自杀意念、自杀计划更多 ${ }^{[64]}$. 抑郁症发作受 多种复杂因素影响. 此外, 所在省份信息也应被纳人
考察范围内, 语音往往具有显著的地域特色. 例如, 南方方言相比北方方言通常有更多的声调变化 ${ }^{[65]}$. 我们未能在语音数据收集过程中对协变量进行很好 的匹配. 排除潜在混淆变量的影响将有助于进一步 理解抑有症, 对结果做出更加全面的解释.

虽然在该样本中同时涵盖了男性与女性群体, 但是由于样本数量有限, 未能对两种性别进行单独 考察. 男性、女性在语音上本就存在这较大的差别, 未来研究应该单独考虑男性与女性各自的语音特性.

而且本研究只考虑了抑郁症患者和健康人之间 的区别. 在临床诊断中, 抑郁症往往会与双相障碍等 其他精神障碍相混淆, 从而导致诊断的困难. 未来研 究有必要划分更加细致的层次, 全面考察语音预测 抑郁症在不同人群(如躯体疾病患者、其他精神障碍 患者)的区分效果. 从而获得更加严谨的结论.

鉴于本研究验证了语音对抑郁症具有跨情景的 预测作用, 未来研究可以尝试采用更加有效的处理 高维数据的方法, 寻找对语音具有预测作用的最佳 特征, 提高模型的泛化能力. 例如, 对特征进行降 维. 有研究尝试采用瓶颈特征选择的方法来篮选特 征, 借助于多重感知器、引人瓶颈层来降低特征的维 度. 该方法对口音、环境噪声、设备差异等具有较好 的鲁棒性 ${ }^{[66]}$. 另有更加复杂的人工神经网络等算法, 如时间递归神经网络 (long short-term memory)算 法 $^{[67]}$, 该方法能够智能化地、全面而非孤立地考虑前 后信息之间的关联，做出更加合理的预测.

\section{4 结论}

本研究采用机器学习的方法, 在不同实验条件 下, 通过对高维语音数据建立是否抑郁的二分类的 预测模型, 说明了语音作为临床抑郁症快速识别与 诊断的工具的意义与价值. 鉴于本研究的结果和存 在的不足, 未来研究可以一方面从与抑郁症存在显 著关联的语音特征人手, 进行更加精确的定位和探 讨; 另一方面, 可以借助更加精巧的计算机分类算 法, 并进行有效的重复性验证, 提高模型的精度和泛 化能力, 增强结果的可推广性.

\section{参考文献}

1 Lépine J P, Briley M. The increasing burden of depression. Neuropsychiatr Dis Treat, 2011, 7: 3

2 Mitchell A J, Vaze A, Rao S. Clinical diagnosis of depression in primary care: A meta-analysis. Lancet, 2009, 374: 609-619 
3 Schumann I, Schneider A, Kantert C, et al. Physicians' attitudes, diagnostic process and barriers regarding depression diagnosis in primary care: A systematic review of qualitative studies. Fam Pract, 2011, 29: 255-263

4 Inoue T, Inagaki Y, Kimura T, et al. Prevalence and predictors of bipolar disorders in patients with a major depressive episode: The Japanese epidemiological trial with latest measure of bipolar disorder (JET-LMBP). J Affect Disord, 2015, 174: 535-541

5 Vos T, Allen C, Arora M, et al. Global, regional, and national incidence, prevalence, and years lived with disability for 310 diseases and injuries, 1990-2015: A systematic analysis for the Global Burden of Disease Study 2015. Lancet, 2016, 388: 1545-1602

6 Mundt J C, Snyder P J, Cannizzaro M S, et al. Voice acoustic measures of depression severity and treatment response collected via interactive voice response (IVR) technology. J Neurolinguist, 2007, 20: 50-64

7 Mundt J C, Vogel A P, Feltner D E, et al. Vocal acoustic biomarkers of depression severity and treatment response. Biol Psychiatry, 2012, 72: 580-587

8 Cannizzaro M, Harel B, Reilly N, et al. Voice acoustical measurement of the severity of major depression. Brain Cogn, 2004, 56: 30-35

9 France D J, Shiavi R G, Silverman S, et al. Acoustical properties of speech as indicators of depression and suicidal risk. IEEE T Bio-Med Eng, 2000, 47: 829-837

10 Scherer S, Stratou G, Lucas G, et al. Automatic audiovisual behavior descriptors for psychological disorder analysis. Image Vision Comput, 2014, 32: 648-658

11 Stassen H H. Speaking behavior and voice sound characteristics in depressive patients during recovery. J Psychiat Res, 1993, 27: 289-307

12 Tolkmitt F, Helfrich H, Standke R, et al. Vocal indicators of psychiatric treatment effects in depressives and schizophrenics. J Commun Disord, 1982, 15: 209-222

13 Scherer K R, Sundberg J, Fantini B, et al. The expression of emotion in the singing voice: Acoustic patterns in vocal performance. J Acoust Soc Am, 2017, 142: 1805-1815

14 Moses P J. The Voice of Neurosis. Oxford: Grune \& Stratton, 1954. 5

15 Ostwald P F. Soundmaking. The Acoustic Communication of Emotion. Oxford: Charles C Thomas, 1963. 12

16 Cohen A S, Najolia G M, Kim Y, et al. On the boundaries of blunt affect/alogia across severe mental illness: Implications for research domain criteria. Schizophr Res, 2012, 140: 41-45

17 Covington M A, Lunden S L A, Cristofaro S L, et al. Phonetic measures of reduced tongue movement correlate with negative symptom severity in hospitalized patients with first-episode schizophrenia-spectrum disorders. Schizophr Res, 2012, 142: 93-95

18 Darby J K, Hollien H. Vocal and speech patterns of depressive patients. Folia Phoniatr Logo, 1977, 29: 279-291

19 Szabadi E, Bradshaw C M, Besson J A. Elongation of pause-time in speech: A simple, objective measure of motor retardation in depression. Br J Psychiatry, 1976, 129: 592-597

20 Flint A J, Black S E, Campbell-Taylor I, et al. Abnormal speech articulation, psychomotor retardation, and subcortical dysfunction in major depression. J Psychiatry Res, 1993, 27: 309-319

21 Alpert M, Pouget E R, Silva R R. Reflections of depression in acoustic measures of the patient's speech. J Affect Disord, 2001, 66: 59-69

22 Alghowinem S, Goecke R, Epps J, et al. Cross-cultural depression recognition from vocal biomarkers. In: the 17th Annual Conference of the International Speech Communication Association. France: International Speech Communication Association, 2016. 1943-1947

23 Alghowinem S, Goecke R, Wagner M, et al. Detecting depression: A comparison between spontaneous and read speech. In: 2013 IEEE International Conference on Acoustics, Speech and Signal Processing (ICASSP). New York: IEEE, 2013. 7547-7551

24 Mitra V, Shriberg E. Effects of feature type, learning algorithm and speaking style for depression detection from speech. In: 2015 IEEE International Conference on Acoustics, Speech and Signal Processing (ICASSP). New York: IEEE, 2015. 4774-4778

25 Kaya H, Eyben F, Salah A A, et al. CCA based feature selection with application to continuous depression recognition from acoustic speech features. In: 2014 IEEE International Conference on Acoustics, Speech and Signal Processing. New York: IEEE, 2014. 3729-3733

26 Torres J, Saad A, Moore E. Evaluation of objective features for classification of clinical depression in speech by genetic programming. In: Soft Computing in Industrial Applications. Berlin: Springer, 2007. 132-143

27 Torres J, Saad A, Moore E. Application of a GA/Bayesian filter-wrapper feature selection method to classification of clinical depression from speech data. In: Soft Computing in Industrial Applications. Berlin: Springer, 2007. 115-121

28 Péron J, El Tamer S, Grandjean D, et al. Major depressive disorder skews the recognition of emotional prosody. Prog Neuro Psych, 2011, 35: 987-996

29 Sloan D M, Bradley M M, Dimoulas E, et al. Looking at facial expressions: Dysphoria and facial EMG. Biol Psychol, 2002, 60: 79-90

30 Gilboa-Schechtman E, Erhard-Weiss D, Jeczemien P. Interpersonal deficits meet cognitive biases: Memory for facial expressions in depressed and anxious men and women. Psychiatry Res, 2002, 113: 279-293 
31 Stasak B, Epps J, Cummins N, et al. An investigation of emotional speech in depression classification. In: the 17th Annual Conference of the International Speech Communication Association. France : International Speech Communication Association, 2016. 485-489

32 Hergueta T, Baker R, Dunbar G C. The mini-international neuropsychiatric interview (MINI): The development and validation of a structured diagnostic psychiatric interview for DSM-IVand ICD-10. J Clin Psychiatry, 1998, 59(Suppl 20): 2233

33 American Psychiatric Association. Diagnostic and Statistical Manual of Mental Disorders (DSM-5). Valencia: American Psychiatric Publishing, 2013

34 Liu Z, Hu B, Li X, et al. Detecting depression in speech under different speaking styles and emotional valences. In: International Conference on Brain Informatics. Cham: Springer, 2017. 261-271

35 Wang J Y. An Exploratory study on auxiliary diagnosis of depression based on speech (in Chinese). Doctor Dissertation. Beijing: Graduate School of Chinese Academy of Sciences, 2017 [汪静莹. 抑郁症的辅助诊断研究——基于语音特征的探索. 博士学位论文. 北 京: 中国科学院研究生院, 2017]

36 Eyben F, Weninger F, Gross F, et al. Recent developments in opensmile, the munich open-source multimedia feature extractor. In: Proceedings of the 21st ACM international conference on Multimedia. New York: ACM, 2013. 835-838

Dreiseitl S, Ohno-Machado L. Logistic regression and artificial neural network classification models: A methodology review. J Biomed Inform, 2002, 35: 352-359

39 Powers D M. Evaluation: From precision, recall and F-measure to ROC, informedness, markedness and correlation. J Machine Learning Tech, 2011, 2: 37-83

40 Ling C X, Huang J, Zhang H. AUC: A statistically consistent and more discriminating measure than accuracy. In: Proceedings of the 18th International Joint Conference on Artificial Intelligence. San Francisco: Morgan Kaufmann Publishers Inc., 2003. 519-524

41 Cummins N, Epps J, Sethu V, et al. Modeling spectral variability for the classification of depressed speech. Genes Immun, 2013, 1: 857-861

42 Cohn J F, Kruez T S, Matthews I, et al. Detecting depression from facial actions and vocal prosody. Affective Computing and Intelligent Interaction and Workshops, 2009. ACII 2009. In: 3rd International Conference on. New York: IEEE, 2009. 1-7

Ellgring H, Scherer K R. Vocal indicators of mood change in depression. J Nonverbal Behav, 1996, 20: 83-110

Nilsonne A. Speech characteristics as indicators of depressive illness. Acta Psychiatr Scan, 1988, 77: 253-263

Savitz J, Drevets W C. Bipolar and major depressive disorder: Neuroimaging the developmental-degenerative divide. Neurosci Biobehav Rev, 2009, 33: 699-771

Peluso M A, Glahn D C, Matsuo K, et al. Amygdala hyperactivation in untreated depressed individuals. Psychiatry Res, 2009, 173: 158-161

47 Siegle G J, Steinhauer S R, Thase M E, et al. Can't shake that feeling: Event-related fMRI assessment of sustained amygdala activity in response to emotional information in depressed individuals. Biol Psychiatry, 2002, 51: 693-707

48 Thomas K M, Drevets W C, Dahl R E, et al. Amygdala response to fearful faces in anxious and depressed children. Arch Gen Psychiatry, 2001, 58: 1057-1063

49 Epstein J, Pan H, Kocsis J H, et al. Lack of ventral striatal response to positive stimuli in depressed versus normal subjects. Am J Psychiatry, 2006, 163: 1784-1790

50 Fu C H, Williams S C, Cleare A J, et al. Attenuation of the neural response to sad faces in major depression by antidepressant treatment: A prospective, event-related functional magnetic resonance imaging study. Arch Gen Psychiatry, 2004, 61: 877-889

51 Surguladze S, Brammer M J, Keedwell P, et al. A differential pattern of neural response toward sad versus happy facial expressions in major depressive disorder. Biol Psychiatry, 2005, 57: 201-209

52 Grimm S, Beck J, Schuepbach D, et al. Imbalance between left and right dorsolateral prefrontal cortex in major depression is linked to negative emotional judgment: An fMRI study in severe major depressive disorder. Biol Psychiatry, 2008, 63: 369-376

53 Drevets W C. Neuroimaging and neuropathological studies of depression: implications for the cognitive-emotional features of mood disorders. Curr Opin Neurobiol, 2001, 11: 240-249

54 Chan S W, Harmer C J, Goodwin G M, et al. Risk for depression is associated with neural biases in emotional categorisation. Neuropsychologia, 2008, 46: 2896-2903

55 Anand A, Li Y, Wang Y, et al. Activity and connectivity of brain mood regulating circuit in depression: A functional magnetic resonance study. Biol Psychiatry, 2005, 57: 1079-1088

56 Hendin H, Maltsberger J T, Szanto K. The role of intense affective states in signaling a suicide crisis. J Nerv Ment Dis, 2007, 195: 363-368

57 Beck A T. Depression: Clinical, Experimental, and Theoretical Aspects. Philadelphia: University of Pennsylvania Press, 1967

58 Alghowinem S, Goecke R, Wagner M, et al. A comparative study of different classifiers for detecting depression from spontaneous 
speech. In: 2013 IEEE International Conference on Acoustics, Speech and Signal Processing. New York: IEEE, 2013. 8022-8026

59 Porritt L L, Zinser M C, Bachorowski J A, et al. Depression diagnoses and fundamental frequency-based acoustic cues in maternal infant-directed speech. Lang Learn Dev, 2014, 10: 51-67

60 Lever J, Krzywinski M, Altman N. Points of significance: Logistic regression. Nat Methods, 2016, 13: 541-542

61 Alghowinem S, Goecke R, Wagner M, et al. Multimodal depression detection: Fusion analysis of paralinguistic, head pose and eye gaze behaviors. In: IEEE Transactions on Affective Computing. New York: IEEE, 2016

62 Yang F, Li Y, Xie D, et al. Age at onset of major depressive disorder in Han Chinese women: Relationship with clinical features and family history. J Affect Disord, 2011, 135: 89-94

63 Yang F, Zhao H, Wang Z, et al. Age at onset of recurrent major depression in Han Chinese women-A replication study. J Affect Disord, 2014, 157: 72-79

64 Gan Z, Li Y, Xie D, et al. The impact of educational status on the clinical features of major depressive disorder among Chinese women. J Affect Disord, 2012, 136: 988-992

65 Wang D C. Introduction to Linguistics (in Chinese). Shanghai: Shanghai Foreign Language Education Press, 1997. 60-95 [王德春. 语言 学概论. 上海: 上海外语教育出版社, 1997. 60-95]

66 Wang Y, Yang J A, Liu H, et al. Bottleneck feature extraction method based on hierarchical deep sparse belief network (in Chinese). Pattern Recogn Artif Intell, 2015, 28: 173-180 [王一, 杨俊安, 刘辉, 等. 基于层次稀疏 DBN 的瓶颈特征提取方法. 模式识别与人 工智能, 2015, 28: 173-180]

67 Huang K Y, Wu C H, Kuo Y T, et al. Unipolar depression vs. bipolar disorder: An elicitation-based approach to short-term detection of mood disorder. Depression, 2016, 10: 12

\section{补充材料}

补充材料 1 实验使用材料

补充材料 2 结果图片

本文以上补充材料见网络版 csb.scichina.com. 补充材料为作者提供的原始数据, 作者对其学术质量和内容负责. 
Summary for “基于语音的抑郁症识别”

\title{
Depression recognition based on speech analysis
}

\author{
Wei Pan ${ }^{1,2}$, Jingying Wang ${ }^{1,2}$, Tianli Liu ${ }^{3}$, Xiaoqian Liu ${ }^{1}$, Mingming Liu ${ }^{1,2},{\text { Bin } \mathrm{Hu}^{4} \text { \& Tingshao Zhu }}^{1 *}$ \\ ${ }^{1}$ Institute of Psychology, Chinese Academy of Sciences, Beijing 100101, China; \\ ${ }^{2}$ Department of Psychology, University of Chinese Academy of Sciences, Beijing 100049, China; \\ ${ }^{3}$ Institute of Population Research, Peking University, Beijing 100871, China; \\ ${ }^{4}$ School of Information Science and Engineering, Lanzhou University, Lanzhou 730000, China \\ * Corresponding author, E-mail: tszhu@psych.ac.cn
}

Depression is one of the common mental diseases. Patients with depression often have depressed moods such as sadness, guilty, low self-esteem, loss of interest, hypofunction and so on. They suffer from serious emotional problems, unexplained suffering, which has caused enormous losses to individuals, families and society. According to the World Health Organization, there are aproximately 322 million people suffering from depression in the whole world in 2017. While there are about 54 million depressive patients in China.

Depression can be cured effciently. However, due to the complexity of the pathogenesis of depression, clinical diagnosis is accompanied with many difficulties. Firstly, the mental disease, especially depression, are not getting enough attention and even being misinterpreted by other people. Secondly, the depression patients are less willing to ask for help. Thirdly, it is hard to select and dignose the potential depression patients precisely, as well as there are limited medical resource for depression diagnosis.

It is necessary to find a more convenient, objective and efficient way to assist the fast identification of depression. As a relatively objective and easily accessible variable, speech has its potential value. The speech of patient is easy to acquire, and also, it has been proved that the sound of depressed patients have special charcteristics such as slow speech rate, lack of cadence and so on. The purpose of this paper is to explore the relationship between speech and depression by establishing classification models of voice feature and depression prediction. In this research, 3(emotion mood: positive, neutral, negative) $\times 3$ (task type: question answering, text reading, picture description) experimental design was employed, and the voice data was collected from the speech of individuals recorded during different tasks. 103 participants were inculded in this study, including 45 depression patients (age: 23.8-44.6, $M=34.2, S D=10.4$, males=22, females=23) and 58 healthy ones (age: $20.1-41.7, M=30.9, S D=10.8$, males=27, females=31). The former were recruited in the hospital in Beijing Anding Hospital and Huilongguan Hospital, while the latter were recruited by advertisement. All of them were diagnosed by specialist with DSM-IV and MINI interview. All participants did not have substance abuse, substance dependence, personality disorders and other mental diseases, no serious physical illness or suicidal behavior. The education level of subjects are all above the elementary school. 988 Voice features were extracted from the speech data using open SMILE software. Logistic regression, a machine learning method, was used to train the predicting models. Results showed that the precision rate of predicting can reach to $82.9 \%$. Based on machine learning methods, this paper employed voice features to establish predicting models of depression. Results show the speech of depression patients has certain predicting effect, which paves the way for the further identification of depression in a more thorough way.

\section{depression, voice feature, classification algorithm, logistic regression}

doi: 10.1360/N972017-01250 